\title{
Penetrating Traumatic Diaphragm Injuries
}

\author{
Mia DeBarros • Matthew J. Martin
}

Published online: 31 March 2015

(C) Springer International Publishing AG (outside the USA) 2015

\begin{abstract}
Traumatic diaphragm injuries (TDI) are uncommon but can result in major morbidity or mortality if missed. Penetrating thoracoabdominal injuries carry a high risk of TDI, but can also pose a diagnostic dilemma due to their small size and the frequent lack of an associated hernia. Despite advances in imaging, diagnosis without presence of a hernia remains difficult and a high index of suspicion must be maintained. Chest x-ray remains an important tool in early diagnosis, but primarily relies on the presence of a hernia or a discontinuity of the diaphragm, which is not typically seen with penetrating injuries. Computed tomography has a higher sensitivity and specificity and improves preoperative diagnosis, but most TDI are still diagnosed intra-operatively. Minimally invasive modalities allow for both diagnosis and repair of suspected injuries in hemodynamically stable patients while avoiding the morbidity of an open approach. All penetrating diaphragmatic injuries require repair in order to avoid the major morbidity and mortality of a chronic diaphragmatic hernia. The principles of diaphragmatic injury repair are complete reduction of all abdominal contents, lavage and evacuation of any associated hemothorax or gastrointestinal spillage, and watertight, tension-free closure. Most inju-
\end{abstract}

This article is part of the Topical Collection on Penetrating Injuries to Solid Abdominal Viscera

M. DeBarros • M. J. Martin $(\bowtie)$

Department of Surgery, Madigan Army Medical Center, 9040a

Fitzsimmons Drive, Tacoma, WA 98431, USA

e-mail: matthew.j.martin16.mil@mail.mil

M. DeBarros

e-mail: mia.d.debarros.mil@mail.mil

M. J. Martin

Department of Surgery, Legacy Emanuel Medical Center,

Portland, OR, USA ries can be closed primarily, but some may require use of prosthetic material or other advanced reconstructive techniques for larger defects. Mortality remains primarily dependent on the mechanism of injury and the presence and severity of associated injuries, with overall Injury Severity Score serving as an independent predictor of early mortality.

Keywords Trauma $\cdot$ Diaphragm injuries $\cdot$ Blunt trauma . Penetrating trauma $\cdot$ Diaphragmatic hernia .

Thoracoabdominal trauma

\section{Introduction and History}

Proper diaphragm structure and function is crucial for lung expansion, respiration, and maintenance of a pressure differential and anatomic separation between the abdominal and thorax [1]. Although historically traumatic diaphragm injuries (TDI) were rare, their incidence has increased in modern times [2]. In 1541, Sennertus described a TDI with gastric herniation on autopsy after a stab wound. Ambroise Paré reported a similar finding in 1578 in a soldier with colonic strangulation after a missed TDI from a stab wound. Over 300 years later, Riolfi reported the first successful repair of a diaphragmatic hernia, and Walker reported the first repair of a trauma-related hernia in 1900 [3]. The high morbidity and mortality associated with TDI was appreciated after World War I with an increase in strangulated diaphragmatic hernias, and by 1951, Carter published the first comprehensive review on TDI $[4,5]$.

\section{Incidence and Epidemiology}

The incidence of TDI ranges from 0.8 to $8 \%$, but the true incidence is likely higher due to missed or delayed diagnoses [6]. TDI can often be missed even during exploratory surgery; 
with one series demonstrating $14 \%$ of TDI were missed at an initial laparotomy [7]. This is likely due to the lack of a high index of suspicion and the difficulty with direct visualization of the hemi-diaphragms. This is particularly important in penetrating trauma victims, as the injuries are typically smaller and may not be immediately obvious.

TDI is more likely with penetrating wounds compared to blunt trauma, and is highest among thoracoabdominal gunshot wounds (GSW). Despite the fact that penetrating trauma accounts for only 5 to $10 \%$ of admissions at most trauma centers, approximately two-thirds (65-73\%) of all TDI are secondary to penetrating mechanisms $[6,8 \bullet]$. While most blunt TDI are located in the central or posterolateral diaphragm and are related to embryologic weakness, penetrating TDI can occur anywhere on the diaphragm [9].

TDI is most commonly diagnosed on the left side, representing $75 \%$ of cases [2]. Right-sided TDI is less common, with most series reporting rates of 35-49\%. However, right-sided TDI are harder to diagnose due to coverage by the liver, and the true incidence is almost certainly higher than reported. The higher prevalence of left-sided TDI is also associated with the mechanics of penetrating wounds. There are more right-handed assailants and thus a preference for wounding to the left side of the victim. On the right side, the liver may prevent injuries and can mask or seal many smaller injuries. Left-sided TDI have more frequent injuries to associated organs, and have a higher morbidity and mortality risk [8•]. However, the liver should not be considered as reliable protection against a diaphragm injury, and these may present years later with large and complex herniations of the liver and other organs $[10,11]$. Bilateral TDI is an extremely rare occurrence, reported as 2-8\% overall but is seen almost exclusively with blunt mechanisms [12•].

Although penetrating abdominal injury has historically mandated an exploratory laparotomy, there are now multiple series that have validated the safety of selective nonoperative management (SNOM). This is based on the fact that most injuries that require operative repair will manifest immediately or within the first several hours. However, it must be recognized that one exception to this rule is for TDI, and any protocol for SNOM must include evaluation and appropriate intervention for TDI. This is particularly true for any GSW in the thoracoabdominal region, or that has injured a solid organ adjacent to the diaphragm. Among patients who had SNOM of GSW to the liver, $68 \%$ were found to have a TDI [13]. In a similar series of patients with GSW to the kidneys, $18 \%$ had an associated TDI [14].

\section{Key Anatomic Concepts}

Although the diaphragm is commonly thought of as a thin horizontal structure that divides the thoracic and abdominal cavities, it is critical to understand the actual location, shape, 3-dimensional anatomy, tissue composition, and relationship to adjacent structures. The first misconception is that the diaphragm is a flat, horizontally oriented structure. It should be thought of as a 3-dimensional cone, with the apex at the midpoints of each hemi-diaphragm and the base representing the lower attachments to the chest wall. The second misconception is that the diaphragm is a static structure located in a reliably reproducible position. The reality is that it is a highly mobile structure with a large degree of motion during respiration. At maximal inhalation, the diaphragmatic cone inverts inferiorly and the lowest point can reach the inferior costal margins. During maximal exhalation, the dome of the diaphragm is displaced superiorly and can reach the level of the nipples.

The diaphragm should be considered as a large set of paired muscles with circumferential attachments to the thoracoabdominal bony structures including the xiphoid and sternum anteriorly, the ribs and costal cartilage laterally, and the spine posteriorly. The thick muscular character of the diaphragm changes to a thinner white aponeurosis centrally, known as the central tendon. The diaphragm contains three hiatal defects at the T8 to T12 level that allow passage of the vena cava (caval hiatus), the esophagus and vagus nerves (esophageal hiatus), and the aorta (aortic hiatus) [15]. The excellent blood supply makes devascularization an exceedingly rare problem. In contrast, the innervation is entirely from the paired phrenic nerves that run in the mediastinum and then enter the diaphragm antero-medially, where they give off several branches [16]. Injuries to the phrenic nerve or larger branches can occur secondary to the initial trauma or due to iatrogenic injury during surgical repair. If additional incisions on the diaphragm are required, these should be made posterolaterally and in a curvilinear orientation. Radial incisions from central to lateral are the next safest to avoid iatrogenic nerve injury. During surgical repair, large "en-masse" suture bites should be avoided and care should be taken in the antero-medial quadrant of each hemi-diaphragm [16].

Another anatomic factor that should be appreciated is the unpredictable path that the penetrating object(s) can take. Trauma victims are rarely shot or stabbed in a normal anatomic position, and this can result in an unexpected and unreliable relationship between the external wounds and the resultant injured structures. Missiles can also be redirected or break into multiple fragments after striking bone. However, projectiles still must obey the laws of physics, and therefore, attempts to delineate entrance and exit wounds and estimate the missile trajectory can be useful in guiding the diagnostic workup or interventions.

The final anatomic concept that is critical is the relationship of the diaphragms to surrounding structures and organs. These relationships are highlighted in Table 1, and injury to the diaphragm should prompt evaluation of the associated organs and 
Table 1 Anatomic areas of the diaphragm and the closely associated organs and structures at risk of injury

\begin{tabular}{|c|c|c|c|}
\hline & \multicolumn{3}{|c|}{ Anatomic region of diaphragm } \\
\hline & Right & Central & Left \\
\hline Solid organs & $\begin{array}{l}\text { Heart } \\
\text { Right lower lobe lung } \\
\text { Right lobe liver } \\
\text { Right kidney } \\
\text { Right adrenal gland }\end{array}$ & $\begin{array}{l}\text { Heart } \\
\text { Right/left lung } \\
\text { Central liver }\end{array}$ & $\begin{array}{l}\text { Heart } \\
\text { Left lower lobe lung } \\
\text { Spleen/pancreas } \\
\text { Left kidney } \\
\text { Left adrenal gland }\end{array}$ \\
\hline Hollow viscus & $\begin{array}{l}\text { Hepatic flexure colon } \\
\text { Gallbladder }\end{array}$ & $\begin{array}{l}\text { Esophagus } \\
\text { Stomach } \\
\text { Small intestine }\end{array}$ & $\begin{array}{l}\text { Stomach } \\
\text { Splenic flexure colon } \\
\text { Small intestine }\end{array}$ \\
\hline Vessels, nerves & $\begin{array}{l}\text { Hepatic veins } \\
\text { Vena cava } \\
\text { Right phrenic nerve }\end{array}$ & $\begin{array}{l}\text { Aorta } \\
\text { Celiac vessels } \\
\text { Vena cava } \\
\text { Azygous vein } \\
\text { Vagus nerves }\end{array}$ & $\begin{array}{l}\text { Splenic artery } \\
\text { Splenic vein } \\
\text { Left phrenic nerve }\end{array}$ \\
\hline Other & $\begin{array}{l}\text { Right ribs } \\
\text { Right scapula }\end{array}$ & $\begin{array}{l}\text { Sternum/xiphoid } \\
\text { Spine/spinal cord }\end{array}$ & $\begin{array}{l}\text { Left ribs } \\
\text { Left scapula }\end{array}$ \\
\hline
\end{tabular}

structures, and vice-versa. Arguably, the most important of these structures is the heart, which rests on the central tendon of the diaphragm and should be of primary concerns in any penetrating thoracoabdominal wound. On the left, the diaphragm is closely opposed to the lower lobe of the lung, the stomach, the kidney, and the spleen/pancreas. On the right, the diaphragm lies adjacent to the lower lobe of the lung, the right liver, and the kidney. The central portion of the diaphragm and the crural openings are intimately associated with the vena cava, aorta, and esophagus in addition to the heart. Although almost any mobile abdominal organ can be present in a traumatic diaphragmatic hernia, the most commonly herniated organs are the stomach (40-50\%), spleen (20-30\%), and bowel (10-20\%) [2, 17, 18$]$.

\section{Diagnosis/Work-up}

The most important point in diagnosing a TDI is that there is no imaging study that will reliably identify (or exclude) the injury in the absence of a diaphragmatic hernia. Penetrating TDI is typically small, with over $80 \%$ being less than $2 \mathrm{~cm}$ [19]. There is also a misperception that only anterior wounds carry a risk of diaphragm injury, while in reality, posterior wounds can just as readily cause TDI. All patients with a penetrating injury to the thoracoabdominal area, or remote ones that entered or crossed this area, should be suspected of having TDI. Signs and symptoms of TDI are often associated with acute herniation of abdominal contents, and patients may report shoulder pain, epigastric pain, vomiting, or shortness of breath. On physical exam, a classic but uncommon finding is the presence of bowel sounds in the chest. The absence of breath sounds is also suggestive (but not specific) of diaphragm injury and can be due to the hernia contents, a co-existing pneumo- or hemothorax, or direct lung injury.

Imaging remains crucial in the early diagnosis of TDI and to identifying other life-threatening injuries. The chest $\mathrm{x}$-ray (CXR) is particularly critical in the patient with a penetrating thoracoabdominal injury, and our preference is to have the CXR plate pre-positioned and to rapidly obtain the CXR in the upright position. This may identify signs of TDI, but is also highly reliable for identifying the majority of lifethreatening pathologies in the chest. The diagnosis can be made in $90 \%$ of patients if there is a hernia present, but without it, the detection rate drops to $23-30 \%$ [18]. The classic findings are an air fluid level or a gastric tube coiled in the left chest, indicating a diaphragmatic hernia. However, only $10-20 \%$ of penetrating TDI present with herniation [20, $21 \bullet, 22]$. Findings associated with TDI without hernia on CXR may be absent, or may consist of obscured diaphragmatic shadow, elevated hemidiaphragm, mediastinal shift away from the injury, and irregular diaphragmatic contour. The differential diagnoses associated with these findings include pneumothorax, hemothorax, pulmonary contusions, and atelectasis, all of which are very common with penetrating trauma. The diagnostic yield may be improved by performing serial CXR imaging if the patient is stable or if CT imaging is not available [23, 24].

In stable patients, computed tomography $(\mathrm{CT})$ remains one of the most useful diagnostic tools. This modality allows for possible rapid detection of a TDI and delineation of other associated injuries. Traditional signs of a TDI include direct visualization of the injury, non-visualization of the diaphragm, and herniation of abdominal viscera into the thorax. Additional indirect signs have been described, including the hump sign, the collar sign, the dependent viscera sign, and peridiaphragmatic contrast extravasation [24]. CT has a reported 
sensitivity of $14-61 \%$ and specificity of $76-99 \%$, particularly if a hernia is present [22]. The sensitivity and specificity improve to 77 and $98 \%$, respectively, with the use of modern multi-detector CT, but these studies include both blunt and penetrating injuries. Up to $90 \%$ sensitivity is reported with blunt TDI, but this decreases to $8-60 \%$ in penetrating TDI $[25,26 \cdot]$. For penetrating TDI, the most important indirect sign on CT scan is the presence of contiguous injuries to structures on both sides of the diaphragm, most commonly a hemothorax or lung injury coupled with a liver injury (rightside) or spleen/gastric injury (left side) [21•]. CT reconstruction of the missile tract for firearm injuries can also be helpful for assessing whether the trajectory is suspicious for traversing the diaphragm $[13,27]$.

Although CXR or CT scan may provide an immediate diagnosis if there is clear herniation present, there are several scenarios where they can be misleading or unreliable [28]. The first is when the stomach is herniated but is full of either liquid or solid contents, and the imaging findings can easily be mistaken for a hemothorax [28]. There are anecdotal reports of chest tubes being mistakenly placed into the herniated stomach or bowel, although this can be avoided by careful technique in placing the chest tube. The second scenario that can result in a delay in diagnosis is with patients who are intubated and on mechanical ventilation. In this case, there may be no herniation of abdominal contents due to the positive intrathoracic pressure preventing migration of abdominal contents. Once the patient is extubated, herniation may occur hours to days after extubation. Repeat imaging should be performed if there is any clinical suspicion, or with new respiratory distress or chest/abdominal pain.

Other modalities such as ultrasound (US) and magnetic resonance imaging (MRI) are also available to aid in the diagnosis of TDI. Ultrasound is highly operator dependent but may be performed as part of the focused abdominal sonography for trauma (FAST) exam. The exam is extended above the diaphragm to evaluate for hemothorax, assess diaphragm motion, herniation of abdominal viscera or flaps of ruptured diaphragm [24]. This exam is mainly useful in the detection of large hernias, and penetrating injuries are typically not well visualized [8•]. MRI is an excellent modality as the diaphragm can be visualized as a discrete structure. Although MRI in the acute trauma setting is limited, it may be a good option in the stable patient with suspicion for TDI and a negative or equivocal CT scan $[2,29]$. Diagnostic peritoneal lavage (DPL) has largely fallen out of favor due to its lack of specificity and sensitivity and technical difficulty, but may still be used in some instances where FAST or CT scan is not available or practical. A cell count $>1000 \mathrm{RBC} / \mathrm{mm}^{3}$ in the setting of a penetrating thoracoabdominal wound is suggestive of TDI. Lavage fluid instilled into the abdominal cavity that then drains out of a chest tube is a highly reliable diagnostic sign TDI [2]. However, false-negative rates with DPL range from $14-60 \%$ in several case series [30].

Minimally invasive surgery is relatively new to the field of trauma, but with improved equipment and increasing confidence among surgeons, it is quickly becoming a more useful modality. Laparoscopy or thoracoscopy can allow for simultaneous diagnosis and definitive treatment of TDI. These injuries have typically gone undiagnosed if the patient does not have other indications for surgical exploration, with a significant incidence of late or chronic diaphragmatic hernia [31-33]. Over the past two decades, the trauma community has recognized the high incidence of missed diaphragm injuries following penetrating thoracoabdominal trauma. Some series have reported up to a $40 \%$ incidence of TDI with penetrating trauma to the left thoraco-abdominal region, many of which are clinically silent $[2,20,26 \bullet]$.

A 1997 study by Murray and colleagues examined the results using an aggressive TDI protocol for all patients with penetrating thoracoabdominal injuries with either laparotomy or laparoscopy [20]. They found an incidence of TDI of $42 \%$, and most importantly, they identified TDI on laparoscopy in $26 \%$ of patients who had no clinical signs or symptoms. They recommended laparoscopy in all patients at risk for penetrating TDI who do not undergo exploratory surgery for other indications, regardless of imaging or exam findings, and we concur with this approach. Another series of 34 asymptomatic patients found occult TDI in $20 \%$ using laparoscopy, for an overall sensitivity of $88 \%$ and specificity of $100 \%$ [34]. One caveat to this approach is that laparoscopy should be delayed for 12-24 h to allow time for serial clinical exams or repeat imaging to rule out the presence of a missed or occult hollow viscus injury. Although most penetrating TDI can be diagnosed and repaired via laparoscopy, there is a role for thoracoscopy as an alternative in select patients. In one series of thoracoabdominal stab wounds, video-assisted thoracoscopic surgery (VATS) identified TDI in $40 \%$, although all identified injuries were subsequently repaired via laparotomy [35]. Multiple other series have demonstrated that VATS can be used as an accurate diagnostic study or to both diagnose and repair TDI safely and effectively [2, 33, 36, 37]. This also has the added benefit of simultaneously treating any co-existing thoracic pathology such as a persistent or retained hemothorax, and providing direct visualization for proper placement of chest tubes [38-40]. It is important to note that many centers and previous publications have specifically focused on left-sided penetrating thoracoabdominal injuries, and recommend aggressive screening only for left-sided wounds. We disagree with this approach, as right-sided penetrating mechanisms can certainly cause diaphragm injury and several series have demonstrated significant rates of TDI with primarily right-sided wounds. Our recommendation is for diagnostic laparoscopy as the standard modality for patients with penetrating thoracoabdominal trauma (on either side) to evaluate 
for TDI. However, VATS is a reliable alternative for diagnosis and simultaneous treatment of any co-existing thoracic pathology. We recommend this approach in all patients who have a penetrating wound that entered or crossed the anatomic area known as "Murray's Box," characterized as a circumferential band from the nipples/inframammary fold superiorly to the bottom of the costal margins inferiorly (Fig. 1).

\section{Management}

Traditional trauma practice was for open surgical exploration of all penetrating upper abdominal or low thoracic wounds in order to identify and treat TDI as well as injury to other thoracic or abdominal structures [41-44]. This nonselective approach has given way to a more selective policy that includes imaging and clinical observation for patients that do not meet criteria for urgent surgery. While this has resulted in reduced morbidity and mortality from unnecessary surgery, it has had the unanticipated effect of increasing the chances for missed or delayed diagnoses of TDI. Although the primary focus of this review is on the management of acute TDI, all trauma surgeons must also be prepared for the challenges of a chronic diaphragmatic hernia from a previously missed injury [31].

Initial management focuses on basic ATLS priorities and is not changed by the presence of a TDI. Since most TDI do not occur in isolation, life-threatening injuries

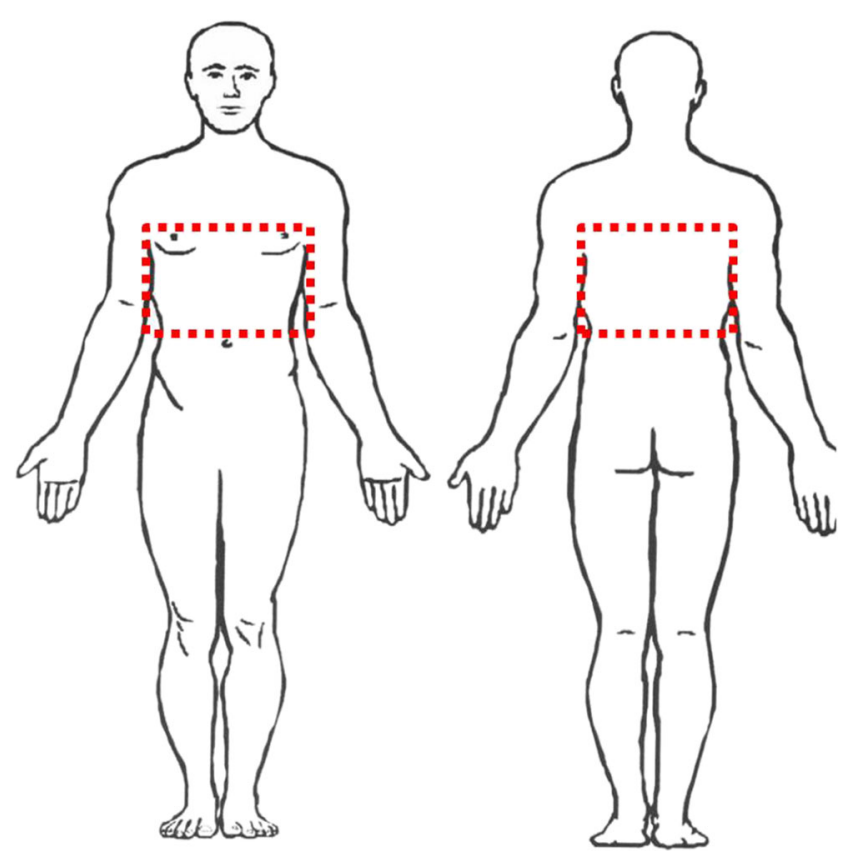

Fig. 1 Anatomic diagram showing the high-risk area (aka "Murray's box") for penetrating diaphragm injuries (red dotted box) both anteriorly and posteriorly. Any penetrating injury in this box, or that crosses or enters this area, should prompt a thorough diagnostic evaluation for diaphragm injury should be addressed first. If the injuries dictate an exploratory laparotomy for other reasons, then this should include a full inspection and palpation of both hemi-diaphragms. This is particularly true in the presence of any injury to closely associated organs or structures as outlined in Table 1. The diaphragm injury itself is rarely an urgent concern, and the workup and management of more critical issues should take precedent. The presence of a TDI will rarely affect oxygenation or ventilation, but associated pulmonary or chest wall injuries may dictate intubation. Bag valve mask ventilation prior to intubation should be minimized if there are known herniated abdominal contents, as this will increase distension and can worsen ischemia.

In practice, the treatment of all TDI is surgical repair with no role for observation even in asymptomatic patients. The assumption has been that the defect will not heal and over time will increase in size. This can result in respiratory compromise, chronic abdominal pain, and strangulation of the herniated organs with significant morbidity and mortality $[23,45,46]$. Although there is no solid human data to contradict this, there is some animal data that suggests that some smaller TDI may heal spontaneously and never become symptomatic [47-49]. However, without any accurate noninvasive way to assess the size and location of the defect, or to predict which will heal spontaneously, all identified injuries should be surgically repaired. Both acute and chronic diaphragmatic injury repair must follow two principles: complete reduction of herniated organs and watertight closure or reconstruction of the diaphragm.

While there is little controversy as to the need for repair, there remain questions as to how best approach the injury. Traditionally, most TDI were identified intra-operatively during exploratory laparotomy [18]. An abdominal approach is advocated because it allows for identification and repair of associated intra-abdominal injuries. The TDI or hernia can also be approached via a thoracotomy, although we would reserve this only for patients who require emergent thoracotomy for associated chest injuries, or in select cases of chronic diaphragmatic hernias. If a thoracic approach is chosen in the acute setting, it should be delayed until the presence of an operative abdominal injury has been definitively ruled out. When an open approach is utilized, the primary perioperative morbidity will be related more to the approach than to the TDI itself. With the rapid advance in minimally invasive techniques and instrumentation, we believe that the majority of these injuries can be safely and effectively repaired laparoscopically or by VATS with a significantly better postoperative morbidity profile.

Minimally invasive surgery has been widely validated in the hemodynamically stable patient with a suspected TDI who does not have other indications for surgery. The initial experience with these techniques used them only for diagnosis, 
with open repair performed if an injury was identified. More recently, minimally invasive repair of these injuries has been well described with excellent outcomes [26•]. Early adopters of this approach cite decreased operative times, lower morbidity and mortality, and decreased length of stay [12•, 26•]. Although specialized equipment and expertise is required, the fact that the vast majority of penetrating TDI are small lacerations makes these procedures relatively straightforward for anyone with strong basic laparoscopic skills. More complex repairs should be referred to someone with more advanced laparoscopic skills, or performed open. Although repair is usually a straightforward primary suture closure, there are several technical tricks to aid in the exposure and repair. Visualization of the TDI can be difficult as the diaphragm is located at the upper apex of the abdomen. A self-retraining retractor will improve exposure and free up the first-assistant to provide manual retraction. The diaphragm is usually mobile, and retraction on each end of the laceration with Allis/ Babcock clamps can bring the injury clearly into view and greatly simplify repair (Fig. 2). Mobilization of the liver may be required to fully visualize right-sided TDI, and the use of steep reverse Trendelenburg position will allow gravity to assist with exposure and retraction.

With minimally invasive repair, the surgeon must anticipate the risk of tension pneumothorax or cardio-respiratory compromise associated with gas insufflation. This can be easily managed by placement of a large-bore IV catheter (14gauge) into the affected thorax to vent gas during the procedure. This can be avoided by use of VATS (does not require gas), lower insufflation pressure, or prophylactic insertion of a needle thoracostomy or chest tube [26•].

The type of repair for TDI is dictated by the extent of injury, patient stability, the surgical approach, and surgeon

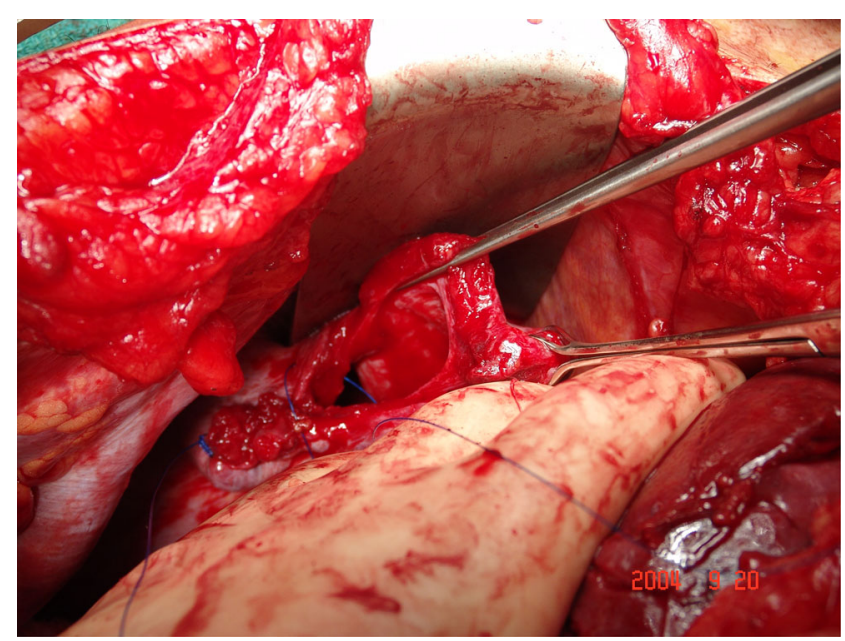

Fig. 2 Intraoperative photograph of a typical penetrating injury to the left diaphragm. The edges of the injury are grasped and elevated using Babcock clamps resulting in improved visualization and exposure for repair with a running non-absorbable suture preference. The American Association for the Surgery of Trauma has developed an organ injury scale, which identifies five grades of injury (Table 2). The majority of penetrating TDI are grade 1 or 2 and amenable to primary suture repair with either absorbable or non-absorbable suture, with no difference in the durability of repair $[8 \bullet, 12 \bullet, 26 \bullet]$. Laparoscopic staplers can also be used to close these defects and appear to provide similar tensile strength [50]. Irregularly shaped defects should be debrided and then repaired with nonabsorbable suture, and although many surgeons recommend techniques such as locking, interrupted horizontal mattress, or figure of eight, there is no evidence that any of these are superior to a simple running suture. Additional considerations include the need for lavage of the thoracic cavity and whether to place a chest tube. Indications for lavage include contamination from aerodigestive injuries or the presence of a significant hemothorax. If visualization is inadequate for lavage or clot evacuation, extending the diaphragm laceration is usually a better option than performing a simultaneous thoracotomy or VATS. Other indications for chest tube placement include pneumo or hemothorax, significant contamination, or the presence of other injuries that require tube drainage. We recommend having a low threshold for placing a chest tube immediately above the TDI repair, as many patients will develop a significant sympathetic pleural effusion from the irritation cause by the injury and repair.

Larger and more complex injuries (grade III to V) are extremely uncommon with civilian firearms, although they may be seen with high-velocity weapons, blast injuries, or shotgun wounds [51-53]. Grade III-IV defects may be mobile enough to repair primarily but often require debridement and a more complex reconstruction. These higher grade defects usually require a prosthetic mesh to effect a tension-free closure. This method is effective in the setting of chronic injury repairs but is limited in the setting of an acute TDI with a contaminated field. Alternative methods in a contaminated field include the use of biologic mesh, vascularized tissue flaps, or a temporary absorbable mesh with plans for a delayed reconstruction [54-57]. A final challenging (although uncommon) situation is diaphragmatic avulsion from its attachments to the chest wall. These injuries can be extremely difficult to repair since

Table 2 American association for the surgery of trauma organ injury scale for diaphragmatic injuries

\begin{tabular}{ll}
\hline Grade $^{\mathrm{a}}$ & Description of injury \\
\hline I & Contusion or hematoma without rupture \\
II & Laceration $<2 \mathrm{~cm}$ \\
III & Laceration $2-10 \mathrm{~cm}$ \\
IV & Laceration $>10 \mathrm{~cm}$ with tissue loss $\leq 25 \mathrm{~cm}^{2}$ \\
V & Laceration with tissue loss $>25 \mathrm{~cm}^{2}$ \\
\hline
\end{tabular}

${ }^{\text {a }}$ Advance one grade for bilateral injuries up to grade III 
the diaphragm retracts and shortens, making reapproximation impossible or prone to failure due to tension. An elegant solution to this is to perform a diaphragmatic transposition, by re-attaching the torn diaphragm edge to the chest wall several rib-spaces higher than normal $[56,58,59]$.

\section{Long-Term Outcomes and Chronic Diaphragmatic Hernia}

Mortality associated with TDI is dependent on the mechanism of injury and is reported as high as $50 \%$ in some case series. A recent multi-center review reported a mortality of $4-15 \%$, with penetrating TDI carrying a significantly lower mortality compared to blunt $[8 \bullet, 12 \bullet, 60]$. The morbidity is almost always due to the type and severity of associated injuries, and rarely related to the TDI itself. A recent study of 237 penetrating trauma victims found no difference in morbidity or mortality between those with and without a TDI [61•]. The most common morbidity related to the TDI repair includes a reactive pleural effusion that may require drainage or can progress to empyema, bleeding into the chest or abdomen, injury to the phrenic nerve, and acute or chronic failure of the repair.

In stark contrast to the low additive morbidity from a diagnosed penetrating TDI, there is significant morbidity and mortality associated with the delayed presentation of missed injuries that present as chronic diaphragmatic hernias. Although the natural history of untreated TDI in humans is not known, it is widely accepted that most injuries will progress to a chronic hernia. The actual incidence of missed TDI is unknown, but is likely much lower with penetrating versus blunt mechanisms since a much higher percentage of these patients undergo surgical exploration $[7,62,63]$. This uncertainty is also due to the lack of an effective non-invasive diagnostic test and the typically long delay between the injury and subsequent diagnosis $[30,31]$. In addition, a missed TDI can carry significant legal liability for the original physician or hospital [64]. One classification of TDI divides it into three categories based on time from original injury. The acute phase occurs from time of injury until apparent recovery. The latent phase includes both symptomatic and asymptomatic patients without the presence of acute obstruction or strangulation. The obstructive phase occurs when the hernia becomes incarcerated and can progress to strangulation, necrosis, and perforation [65]. Chronic hernias are typically diagnosed either as an incidental imaging finding, or when they become large enough to produce symptoms. Although less common, some may remain clinically silent until they present with strangulation and organ ischemia or perforation [31, 33, 58, 66].

The pathophysiology of chronic TDH is related to negative pressure of the thoracic cavity and positive pressure of the abdominal cavity, which pulls and pushes the abdominal viscera up through the defect. There are also radial forces that pull the defect edges apart, resulting in enlargement and displacement of abdominal contents into the chest [67]. Over time, this can lead to pulmonary dysfunction and the potential for incarceration and strangulation of herniated organs. Herniated hollow-viscus organs are more likely to volvulize if the defect is large, while strangulation occurs with smaller defects [31]. The diagnosis is usually readily apparent on radiologic imaging, with CT scans being particularly useful for assessment and operative planning [31].

Once identified, the TDH should be repaired unless the patient's underlying medical co-morbidities prevents operative intervention. The phase in which the patient presents determines whether to proceed with an elective repair (incidental finding on imaging) or urgently (incarceration or strangulation of hollow-viscus organs). The operative approach utilized to perform the repair is controversial and related to both the surgeon's preference and the underlying pathology. Many reports in the literature advocate for an open posterolateral thoracotomy, which allows for good visualization and access to the diaphragm and herniated organs $[68,69]$. A transabdominal approach may be utilized, but may not allow sufficient access to the thorax to safely reduce the hernia and lavage the thoracic cavity. Minimally, invasive approaches (thoracoscopy and laparoscopy) have been reported, but have traditionally been less favored in the chronic TDI and TDH setting $[31,70]$. This was due to concerns about being unable to safely reduce the herniated contents and then perform an adequate repair, but many of these concerns have been disproven or minimized with modern equipment and techniques. We believe that elective repair is almost always possible with a laparoscopic approach, with significantly lower morbidity than either a laparotomy or thoracotomy. Emergent cases of strangulation or even perforation can also be managed with a minimally invasive approach, but rapid conversion to an open approach should be performed if the procedure cannot be quickly and safely accomplished. In select cases where the stomach is the incarcerated organ, reduction or detorsion can be performed endoscopically and maintained with a nasogastric tube, converting an emergent procedure to a semi-elective intervention. Defects that are too large or result in flattening of the diaphragm should be repaired using a prosthetic or biologic mesh to achieve a tension-free repair, or one of the other reconstructive options described previously for grade 3 to 5 defects $[31,55]$.

Morbidity and mortality from chronic TDH is directly related to patient age and comorbidities, and whether there is perforation or ischemia of the hernia contents. Additional morbidity is related to the surgical approach, with an increased incidence of wound complications associated with laparotomy and increased wound and pulmonary complications associated with thoracotomy [31, 71]. There is little debate that an aggressive approach to early diagnosis and repair is warranted in order to avoid 
the devastating outcomes seen with emergent presentations. Patients in the obstructive phase have a reported mortality of up to $80 \%$ and up to a $60 \%$ morbidity rate $[17,30,72]$. These dismal outcomes are in sharp contrast to the exceedingly low morbidity and mortality seen with either initial repair of a promptly diagnosed TDI, or elective surgical repair of a chronic hernia diagnosed in the latent phase [31, 32, 61• , 71].

\section{Conclusion}

Traumatic diaphragmatic injury (TDI) can result in high rates of mortality and morbidity if missed or managed inappropriately. Despite advances in imaging, diagnosis without the presence of a hernia remains difficult and a high index of suspicion must be maintained. All patients with penetrating injuries in the high-risk zone (Fig. 1) should be assumed to have a TDI until proven otherwise. No imaging study is adequately sensitive to identify most TDI unless a hernia is present and diagnostic laparoscopy or VATS should be used liberally. All identified TDI should be surgically repaired by either an open or minimally invasive approach. Most penetrating TDI can be closed primarily, but more complex defects or chronic hernias may require more complex reconstructive options. Outcomes with TDI are primarily related to patient factors and the type and severity of associated injuries. Missed TDI that become chronic diaphragmatic hernias have the potential for major morbidity and mortality due to incarceration and strangulation of hernia contents.

\section{Compliance with Ethics Guidelines}

Conflict of Interest Mia DeBarros and Matthew Martin declare no conflicts of interest.

Human and Animal Rights and Informed Consent This article does not contain any studies with human or animal subjects performed by any of the authors.

Disclaimer The views expressed are those of the author(s) and do not reflect the official policy of the Department of the Army, the Department of Defense or the U.S. Government.

\section{References}

Papers of particular interest, published recently, have been highlighted as:

- Of importance

1. Maish MS. The diaphragm. Surg Clin N Am. 2010;90(5):955-68.

2. Hanna WC, Ferri LE. Acute traumatic diaphragmatic injury. Thorac Surg Clin. 2009;19(4):485-9.
3. Sharma OP, Duffy B. Transdiaphragmatic intercostal hernia: review of the world literature and presentation of a case. J Trauma. 2001;50(6):1140-3.

4. Carter BN, Giuseffi J, Felson B. Traumatic diaphragmatic hernia. Am J Roentgenol Radium Ther. 1951;65(1):56-72.

5. Meyers BF, McCabe CJ. Traumatic diaphragmatic hernia. Occult marker of serious injury. Ann Surg. 1993;218(6):783-90.

6. Lopez PP, Arango J, Gallup TM, et al. Diaphragmatic injuries: what has changed over a 20-year period? Am Surg. 2010;76(5):512-6.

7. Esme H, Solak O, Sahin DA, Sezer M. Blunt and penetrating traumatic ruptures of the diaphragm. Thorac Cardiovasc Surg. 2006;54(5):324-7.

8. Zarour AM, El-Menyar A, Al-Thani H, Scalea TM, Chiu WC. Presentations and outcomes in patients with traumatic diaphragmatic injury: a 15-year experience. J Trauma Acute Care Surg. 2013;74(6):1392-8. quiz 1611. Zarour et al. report the largest single institution series in the current literature of 773 patients with TDI over a 15-year period. The majority (73\%) of these patients were penetrating trauma victims, and most were managed with laparotomy and direct suture repair of the injury. They provide exceptional descriptive data of injury patterns, associated injuries, and outcomes from TDI, including independent predictors of mortality from a multivariate regression model.

9. Dirican A, Yilmaz M, Unal B, Piskin T, Ersan V, Yilmaz S. Acute traumatic diaphragmatic ruptures: a retrospective study of 48 cases. Surg Today. 2011;41(10):1352-6.

10. Boulanger BR, Milzman DP, Rosati C, Rodriguez A. A comparison of right and left blunt traumatic diaphragmatic rupture. J Trauma. 1993;35(2):255-60.

11. Starling SV, Rodrigues Bde L, Martins MP, da Silva MS, Drumond DA. Non operative management of gunshot wounds on the right thoracoabdomen. Rev Col Bras Cir. 2012;39(4):286-94.

12. Ties JS, Peschman JR, Moreno A, et al. Evolution in the management of traumatic diaphragmatic injuries: a multicenter review. J Trauma Acute Care Surg. 2014;76(4):1024-8. Ties et al. retrospectively analyzed a large multicenter cohort of patients with both blunt and penetrating TDI over a 7-year period. They identified a significant increase in the incidence of diagnosed diaphragm injuries among both patient populations although there was little change in the imaging workup. They also found a significant increase in the use of minimally invasive techniques to both diagnose and repair these injuries. This is one of the largest and most comprehensive analyses of TDI in the literature.

13. Navsaria PH, Nicol AJ, Krige JE, Edu S. Selective nonoperative management of liver gunshot injuries. Ann Surg. 2009;249(4):6536.

14. Navsaria PH, Nicol AJ. Selective nonoperative management of kidney gunshot injuries. World J Surg. 2009;33(3):553-7.

15. Nason LK, Walker CM, McNeeley MF, Burivong W, Fligner CL, Godwin JD. Imaging of the diaphragm: anatomy and function. Radiogr Rev Publ Radiol Soc North Am Inc. 2012;32(2):E51-70.

16. Anraku M, Shargall Y. Surgical conditions of the diaphragm: anatomy and physiology. Thorac Surg Clin. 2009;19(4):419-29. 4.

17. Demetriades D, Kakoyiannis S, Parekh D, Hatzitheofilou C. Penetrating injuries of the diaphragm. Br J Surg. 1988;75(8):824-6.

18. Hanna WC, Ferri LE, Fata P, Razek T, Mulder DS. The current status of traumatic diaphragmatic injury: lessons learned from 105 patients over 13 years. Ann Thorac Surg. 2008;85(3):1044-8.

19. Wise L, Connors J, Hwang YH, Anderson C. Traumatic injuries to the diaphragm. J Trauma. 1973;13(11):946-50.

20. Murray JA, Demetriades D, Cornwell 3rd EE, et al. Penetrating left thoracoabdominal trauma: the incidence and clinical presentation of diaphragm injuries. J Trauma. 1997;43(4):624-6.

21. Panda A, Kumar A, Gamanagatti S, Patil A, Kumar S, Gupta A. Traumatic diaphragmatic injury: a review of CT signs and the difference between blunt and penetrating injury. Diagn Interv Radiol. 
2014;20(2):121-8. Panda and colleagues performed a detailed and thorough review of imaging findings associated with TDI, and most importantly they compared the utility of individual diagnostic signs for blunt versus penetrating TDI in the absence of a diaphragmatic hernia. They found that although the standardly reported radiologic signs were useful for blunt injuries, they were mostly unreliable in penetrating trauma. The most reliable sign for penetrating TDI was the presence of contiguous injuries to organs on each side of the diaphragm, which was present in over $80 \%$ of cases.

22. Patlas MN, Leung VA, Romano L, Gagliardi N, Ponticiello G, Scaglione M. Diaphragmatic injuries: why do we struggle to detect them? Radiol Med. 2014;120(1):12-20.

23. Gwely NN. Outcome of blunt diaphragmatic rupture. Analysis of 44 cases. Asian Cardiovasc Thorac Ann. 2010;18(3):240-3.

24. Matsevych OY. Blunt diaphragmatic rupture: four year's experience. Hernia J Hernias Abdom Wall Surg. 2008;12(1):73-8.

25. Hammer MM, Flagg E, Mellnick VM, Cummings KW, Bhalla S, Raptis CA. Computed tomography of blunt and penetrating diaphragmatic injury: sensitivity and inter-observer agreement of CT Signs. Emerg Radiol. 2014;21(2):143-9.

26. Mjoli M, Oosthuizen G, Clarke D, Madiba T. Laparoscopy in the diagnosis and repair of diaphragmatic injuries in left-sided penetrating thoracoabdominal trauma: laparoscopy in trauma. Surg Endosc. 2014;29(3):747-52. Mjoli et al. report a series of 55 patients with penetrating left thoracoabdominal trauma who underwent laparoscopy to diagnose and repair any associated diaphragm injuries. Among the 55 patients, $40 \%$ had a TDI identified, the majority of which were due to stab wounds. The vast majority (96\%) of patients were able to have successful repair of their injury laparoscopically, with no deaths or major morbidity and short overall hospital stays.

27. Oparah SS, Mandal AK. Penetrating gunshot wounds of the chest in civilian practice: experience with 250 consecutive cases. Br J Surg. 1978;65(1):45-8.

28. Sprunt JM, Brown CV, Reifsnyder AC, Shestopalov AV, Ali S, Fielder WD. Computed tomography to diagnose blunt diaphragm injuries: not ready for prime time. Am Surg. 2014;80(11):1124-7.

29. Iochum S, Ludig T, Walter F, Sebbag H, Grosdidier G, Blum AG. Imaging of diaphragmatic injury: a diagnostic challenge? Radiographics Rev Publ Radiol Soc North Am Inc. 2002;22(Spec No):S103-16. discussion S116-108.

30. Payne Jr JH, Yellin AE. Traumatic diaphragmatic hernia. Arch Surg. 1982;117(1):18-24.

31. Blitz M, Louie BE. Chronic traumatic diaphragmatic hernia. Thorac Surg Clin. 2009;19(4):491-500.

32. Celik A, Altinli E, Koksal N, et al. Diagnostic process and management of diaphragmatic injuries: approach in patients with blunt and penetrating trauma. Ulus Travma Acil Cerrahi Der Turk J Trauma Emerg Surg TJTES. 2010;16(4):339-43.

33. Koehler RH, Smith RS. Thoracoscopic repair of missed diaphragmatic injury in penetrating trauma: case report. J Trauma. 1994;36(3):424-7.

34. Friese RS, Coln CE, Gentilello LM. Laparoscopy is sufficient to exclude occult diaphragm injury after penetrating abdominal trauma. J Trauma. 2005;58(4):789-92.

35. Nel JH, Warren BL. Thoracoscopic evaluation of the diaphragm in patients with knife wounds of the left lower chest. Br J Surg. 1994;81(5):713-4

36. Divisi D, Battaglia C, De Berardis B, et al. Video-assisted thoracoscopy in thoracic injury: early or delayed indication? Acta Biomed Atenei Parmensis. 2004;75(3):158-63.

37. Ochsner MG, Rozycki GS, Lucente F, Wherry DC, Champion HR. Prospective evaluation of thoracoscopy for diagnosing diaphragmatic injury in thoracoabdominal trauma: a preliminary report. J Trauma. 1993;34(5):704-9. discussion 709-710.

38. Billeter AT, Druen D, Franklin GA, Smith JW, Wrightson W, Richardson JD. Video-assisted thoracoscopy as an important tool for trauma surgeons: a systematic review. Langenbeck's Arch Surg Dtsch Ges Chir. 2013;398(4):515-23.

39. Ahmad T, Ahmed SW, Soomro NH, Sheikh KA. Thoracoscopic evacuation of retained post-traumatic hemothorax. J Coll Physicians Surg Pak JCPSP. 2013;23(3):234-6.

40. Komatsu T, Neri S, Fuziwara Y, Takahashi Y. Video-assisted thoracoscopic surgery (VATS) for penetrating chest wound: thoracoscopic exploration and removal of a penetrating foreign body. Can J Surg J Can Chir. 2009;52(6):E301-2.

41. Miller L, Bennett Jr EV, Root HD, Trinkle JK, Grover FL. Management of penetrating and blunt diaphragmatic injury. J Trauma. 1984;24(5):403-9.

42. Symbas PN, Vlasis SE, Hatcher Jr C. Blunt and penetrating diaphragmatic injuries with or without herniation of organs into the chest. Ann Thorac Surg. 1986;42(2):158-62.

43. Nicolaou N, Conlan AA. High-velocity missile injuries of the thorax. S Afr Med J Suid-Afrikaanse Tydskrif vir Geneeskunde. 1982;62(10):324-8.

44. Borja AR, Ransdell H. Treatment of thoracoabdominal gunshot wounds in civilian practice. Experience with forty-four cases. Am J Surg. 1971;121(5):580-2.

45. Mihos P, Potaris K, Gakidis J, et al. Traumatic rupture of the diaphragm: experience with 65 patients. Injury. 2003;34(3):169-72.

46. Turhan K, Makay O, Cakan A, et al. Traumatic diaphragmatic rupture: look to see. Eur J Cardiothorac Surg Off J Eur Assoc Cardiothorac Surg. 2008;33(6):1082-5.

47. Gamblin TC, Wall Jr CE, Morgan 3rd JH, Erickson DJ, Dalton ML, Ashley DW. The natural history of untreated penetrating diaphragm injury: an animal model. J Trauma. 2004;57(5):989-92.

48. Shatney $\mathrm{CH}$, Sensaki K, Morgan L. The natural history of stab wounds of the diaphragm: implications for a new management scheme for patients with penetrating thoracoabdominal trauma. Am Surg. 2003;69(6):508-13.

49. Zierold D, Perlstein J, Weidman ER, Wiedeman JE. Penetrating trauma to the diaphragm: natural history and ultrasonographic characteristics of untreated injury in a pig model. Arch Surg. 2001;136(1):32-7.

50. Murray JA, Cornwell 3rd EE, Velmahos GC, et al. Healing of traumatic diaphragm injuries: comparison of laparoscopic versus open techniques in an animal model. J Surg Res. 2001;100(2): 189-91.

51. Bender JS, Lucas CE. Management of close-range shotgun injuries to the chest by diaphragmatic transposition: case reports. J Trauma. 1990;30(12):1581-4.

52. Ivey KM, White CE, Wallum TE, et al. Thoracic injuries in US combat casualties: a 10-year review of operation enduring freedom and Iraqi freedom. J Trauma Acute Care Surg. 2012;73(6 Suppl 5): S514-9.

53. McNamara JJ, Messersmith JK, Dunn RA, Molot MD, Stremple JF. Thoracic injuries in combat casualties in Vietnam. Ann Thorac Surg. 1970;10(5):389-401.

54. Frye WA, Cogbill TH, Patel NY. Diaphragmatic transposition: an elegant procedure for large traumatic chest wall defects. J Trauma. 2005;59(6):1507-9.

55. Al-Nouri O, Hartman B, Freedman R, Thomas C, Esposito T. Diaphragmatic rupture: is management with biological mesh feasible? Int J Surg Case Rep. 2012;3(8):349-53.

56. Muysoms FE, Cathenis KK, Hamerlijnck RP, Claeys DA. Laparoscopic repair of iatrogenic diaphragmatic hernias after sternectomy and pedicled omentoplasty. Hernia J Hernias Abdom Wall Surg. 2009;13(6):617-23.

57. Edington HD, Evans S, Sindelar WF. Reconstruction of a functional hemidiaphragm with use of omentum and latissimus dorsi flaps. Surgery. 1989;105(3):442-5. 
58. Kaths M, Ebert M, Galle PR, Keilmann A, Lang H, Gockel I. Late consequences of traumatic rupture of the diaphragm. Thorac Cardiovasc Surg. 2013;61(3):267-9.

59. Feofilov GL. Transposition of the diaphragm in an operation for a large irreducible diaphragmatic hernia. Vestn Khir Im I I Grek. 1984;133(9):60-2.

60. Williams M, Carlin AM, Tyburski JG, et al. Predictors of mortality in patients with traumatic diaphragmatic rupture and associated thoracic and/or abdominal injuries. Am Surg. 2004;70(2):157-62. discussion 162-153.

61. Ozogul B, Kisaoglu A, Ozturk G, et al. Does a penetrating diaphragm injury have an effect on morbidity and mortality? Ulus Travma Acil Cerrahi Derg Turk J Trauma Emerg Surg TJTES. 2013;19(1):45-8. Ozogul et al. analyzed a series of 237 patients with penetrating trauma to the abdomen over a 15-year period. They compared the 60 patients with a diaphragm injury to 177 without TDI. Although the overall complication rates were high, there was no difference between those with a TDI (47\%) versus those without (50\%). Mortality was also similar at $7 \%$ in the TDI group versus $10 \%$ without a TDI. In addition to providing thorough epidemiologic descriptive data for the TDI cohort, they demonstrated that there appeared to be no added morbidity or mortality associated with the presence of a diaphragm injury.

62. Plurad DS, Nielsen JS, Hancock J, et al. Concomitant rib fractures and minor liver or spleen injuries in blunt trauma: what is the potential for missed diaphragmatic injuries? Am Surg. 2010;76(4): $380-4$.

63. Sangster G, Ventura VP, Carbo A, Gates T, Garayburu J, D'Agostino H. Diaphragmatic rupture: a frequently missed injury in blunt thoracoabdominal trauma patients. Emerg Radiol. 2007;13(5):225-30.

64. Fabian W, Reimer H. Missed traumatic diaphragmatic rupture-a legal insurance problem. Versicherungsmedizin Herausgegeben von Verband der Lebensversicherungs-Unternehmen $\mathrm{eV}$ und Verband der Privaten Krankenversicherung eV. 1991;43(4):122-5.

65. Grimes OF. Traumatic injuries of the diaphragm. Diaphragmatic hernia. Am J Surg. 1974;128(2):175-81.

66. Baca B, Karahasanoglu T, Saribeyoglu K, Arica P, Kol E. Late complication of diaphragmatic gunshot injury: appendix perforation due to colon incarceration. Ulus Travma Acil Cerrahi Derg Turk J Trauma Emerg Surg TJTES. 2007;13(1):70-3.

67. Reber PU, Schmied B, Seiler CA, Baer HU, Patel AG, Buchler MW. Missed diaphragmatic injuries and their long-term sequelae. J Trauma. 1998;44(1):183-8.

68. Kanakis MA, Misthos P, Alexiou K, Karanikas I, Lioulias A. Left thoracotomy utilizing splenectomy in blunt thoracic injury: an alternative surgical approach. Int J Surg Case Rep. 2013;4(4):393-5.

69. Williams RS. Traumatic rupture of the diaphragm. Med J Aust. 1982;1(5):208-11.

70. Amini A, Latifi R. Laparoscopic-assisted minithoracotomy for repair of diaphragmatic penetrating trauma. Surg Laparosc Endosc Percutan Tech. 2013;23(4):406-9.

71. Brasel KJ, Borgstrom DC, Meyer P, Weigelt JA. Predictors of outcome in blunt diaphragm rupture. J Trauma. 1996;41(3):484-7.

72. Degiannis E, Levy RD, Sofianos C, Potokar T, Florizoone MG, Saadia R. Diaphragmatic herniation after penetrating trauma. Br J Surg. 1996;83(1):88-91. 